\title{
The Gut Microbiome of Exudivorous Wild and non-Wild Marmosets
}

\author{
Joanna Malukiewicz ${ }^{1,2, *}$, Reed A. Cartwright ${ }^{3}$, Jorge A. Dergam ${ }^{4}$, Claudia S. Igayara ${ }^{5}$, \\ Sharon Kessler, Silvia B. Moreira ${ }^{7}$, Leanne T. Nash ${ }^{8}$, Patricia A. Nicola ${ }^{9}$, Luiz C.M. \\ Pereira $^{10}$, Alcides Pissinatti ${ }^{7}$, Carlos R. Ruiz-Miranda ${ }^{11}$, Andrew T. Ozga ${ }^{13,14}$, Adriana A. \\ Quirino $^{10}$, Christian Roos ${ }^{1,14}$, Daniel L. Silva ${ }^{15}$, Anne C. Stone ${ }^{8,12,16}$, and Adriana D. \\ Grativol $^{11}$
}

\author{
${ }^{1}$ Primate Genetics Laboratory, German Primate Center, Leibniz Institute for Primate Research, Goettingen, 37077, \\ Germany \\ ${ }^{2}$ Instituto de Medicina Tropical de São Paulo, Universidade de São Paulo, São Paulo, SP, 05403-000, Brazil \\ ${ }^{3}$ School of Life Sciences and The Biodesign Institute, Arizona State University, Tempe, AZ, 85881, USA \\ ${ }^{5}$ Guarulhos Municipal Zoo, Guarulhos, SP, 07081-120, Brazil \\ ${ }^{6}$ Department of Psychology, Faculty of Natural Sciences, University of Stirling, Stirling, FK9 4LA, Scotland \\ ${ }^{7}$ Centro de Primatologia do Rio de Janeiro, Guapimirim, RJ, 25940-000, Brazil \\ ${ }^{8}$ School of Human Evolution and Social Change, Arizona State University, Tempe, AZ, 85281, USA \\ ${ }^{9}$ Programa de Pos-Graduacao Ciencias da Saude e Biologicas, Universidade Federal do Vale do Sao Francisco, \\ Petrolina, PE, 56300-000, Brazil \\ ${ }^{10}$ Centro de Conservacao e Manejo de Fauna da Caatinga, Universidade Federal do Vale do, Sao Francisco, \\ Petrolina, PE, 56300-000, Brazil \\ ${ }^{11}$ Laboratorio das Ciencias Ambientais, Centro de Biociencias e Biotecnologia,Universidade Estadual do Norte \\ Fluminense, Campos dos Goytacazes RJ, 28013-602, Brazil \\ ${ }^{12}$ Center for Evolution and Medicine, Arizona State University, Tempe, Arizona, 85281, USA \\ ${ }^{13} \mathrm{Halmos}$ College of Arts and Sciences, Nova Southeastern University, Fort Lauderdale, FL, 33004, USA \\ ${ }^{14}$ Gene Bank of Primates, German Primate Research Center, Leibniz Institute for Primate Research, Goettingen, \\ 37707, Germany \\ ${ }^{15}$ Nucleo de Pesquisas em Ciencias Biologicas - NUPEB, Federal University of Ouro Preto, Ouro Preto, MG, \\ 35400-000, Brazil \\ ${ }^{16}$ USA Institute of Human Origins, Arizona State University, Tempe, Arizona, 85281, USA \\ *jmalukie@gmail.com
}

\section{ABSTRACT}

Among mammals, captive dietary specialists are prone to gastrointestinal distress and suffer the greatest gut microbiome diversity losses relative to wild individuals. Less is known about the gut microbiome of mammalian exudivores, which eat plant exudates, and increased knowledge could improve their management in the wild and captivity. Marmosets represent key mammalian exudivores that in captivity develop gastrointestinal distress symptoms. Thus, we studied Callithrix gut microbiome composition and predictive function through bacterial 16S ribosomal RNA V4 region sequencing from 59 wild and non-wild Callithrix across four species and their hybrids. Host environment had a stronger effect on the gut microbiome than host taxon. Wild Callithrix gut microbiomes were enriched for Bifidobacterium, which process host-indigestible carbohydrates. Captive marmoset guts were enriched for Enterobacteriaceae, a family containing pathogenic bacteria. While gut microbiome function was similar across marmosets, Enterobacteriaceae seem to carry out most microbiome gut functions in captivity. More diverse bacteria seems to perform gut functions in wild marmosets, with Bifidobacterium being important for gut carbohydrate metabolism. Non-wild marmosets showed gut microbiome composition aspects seen in human gastrointestinal diseases. Thus, captivity may perturb the exudivore gut microbiome, which raises implications for captive animal welfare and calls for husbandry modifications. 


\section{Introduction}

The mammalian gut microbiome plays an important role in host physiology ${ }^{1,2}$, and microbiome dysbiosis is thought to negatively impact host health ${ }^{3-5}$. Closely related mammals share similar gut microbiome composition (GMC), which is usually enriched for bacteria associated with the main macronutrients of a host's feeding strategy ${ }^{6,7}$. Yet, environmental factors significantly alter individual host microbiomes, as evidenced by differences in microbiome composition between wild and captive conspecifics ${ }^{8,9}$. Gut microbiome studies of captive and wild mammals show that non-human primates (NHPs) experience relatively large losses of native gut microbiome diversity in captivity compared to the wild ${ }^{5,8}$. Additionally, folivorous (leaf-eating) NHPs are especially prone to GI problems in captivity ${ }^{10}$, and among humans and NHPs dysbiosis in GMC has been tied to gastro-intestinal (GI) diseases ${ }^{4,10,11}$.

Some mammals are exudivorous (exudate-eating) ${ }^{12}$, particularly primates ${ }^{13}$, but their gut microbomes remains relatively less studied. However, Callithrix marmosets, obligate NHP exudivores, are excellent models to study exudivore gut microbiomes. Callithrix species are endemic to Brazil and in the wild nutritionally exploit hard to digest oligosaccharides of tree gums or hardened saps that require fermentation for digestion ${ }^{14,15}$. Marmosets are regularly maintained in captivity as biomedical research models, for captive breeding of endangered C. aurita, and due to illegal pet trafficking ${ }^{16}$. In captivity, marmosets commonly develop symptoms of GI distress like chronic diarrhea, chronic enteritis, and chronic colitis without clear pathogenesis ${ }^{17,18}$. As mammalian dietary specialists, factors within captive environment such as diet may cause gut microbiome dysbiosis in Callithrix, which in turn may be implicated in the pathogenesis of GI distress.

A necessary first step towards understanding diseased GMC profiles is defining baseline GMC variation and function of non-diseased individuals ${ }^{2}$. Thus, comparing the gut microbiome of wild and captive conspecifics is an important step for such approaches. Most Callithrix gut microbiome studies have focused on captive $C$. jacchus to identify specific bacterial strains and on how life history, social, or laboratory conditions affect the $\mathrm{GMC}^{18}$. A review of these studies suggests that there may be an association between GI distress and gut microbiome dysbiosis in Callithrix ${ }^{18}$. Here, we determine gut microbiome profiles of Callithrix sampled in and out of captivity throughout Brazil. We applied 16S ribosomal RNA (rRNA) V4 region amplicon sequencing of Callithrix gut microbiota, and investigated GMC and gut microbiome predictive functional profiles. Anal swabs were sampled from healthy 59 individuals of four species and three hybrid types (Table 1) from the wild and Brazilian captive facilities (Figure 1). We hypothesize that: (1) Similar taxa of bacterial are found in the gut of different Callithrix taxa; (2) captive Callithrix have less diverse GMC than wild Callithrix; and (3) Callithrix GMC and predictive functional profiles are strongly biased toward carbohydrate metabolism.

\section{Results}

We collected anal swabs from 59 adult marmosets in both the wild and captive facilities located across Brazil (Figure 1, Table 1 and Supplementary Table 1). Veterinary clinical evaluations during sample collection deemed all individuals as healthy. Host taxon identification followed previously published phenotype descriptions ${ }^{19,20}$ and personal observations by JM and CSI. Hosts were also classified by their environment as wild (captured as free-range individuals), translocated (born wild but later put into captivity), or captive (born and raised in captivity). We amplified the V4 16s rRNA region with PCR and sequenced the amplicons on the Illumina MiSeq. After initial processing and filtering in Qiime2-2021.221, a total of 10,902,292 sequence reads was obtained with an average of 201,894 (124389.64 +/- SD) reads per sample. After quality filtering, 8,885,656 reads remained with an average 164,549.19 (99,524.230 +/- SD) reads per sample. Afterward, merging of paired-end sequences produced 8,191,034 reads, with an average of 151,685.81 (91,568.49 +/- SD) reads per sample. This information is detailed in Supplementary Table 2 .

Table 1. Host count distribution by taxon, hybrid status, and environment categories.

\begin{tabular}{|l|l|l|l|l|}
\hline Classification & Taxon & Captive & Translocated & Wild \\
\hline C. aurita & 10 & 5 & 3 & 2 \\
\hline C. geoffroyi & 4 & 3 & 0 & 1 \\
\hline C. jacchus & 9 & 9 & 0 & 0 \\
\hline C. penicillata & 7 & 6 & 1 & 0 \\
\hline C. jacchus $x$ C. penicillata & 23 & 1 & 22 & 5 \\
\hline C. penicillata $x$ C. geoffroyi & 5 & 0 & 0 & 0 \\
\hline C. aurita $x$ Callithrix sp. & 1 & 0 & 1 & 0 \\
\hline Total & 59 & 24 & 27 & 8 \\
\hline
\end{tabular}




\section{Diversity of Callithrix Gut Microbiome Bacterial Taxa}

Callithrix alpha diversity analyses were initially carried out in the MicrobiomeAnalyst ${ }^{22}$ Marker-gene Data Profiling (MDP) module where we further filtered and normalized the data. Results of data rarefaction in the MDP module are shown in Figure 2. For alpha diversity, we first calculated separate Shannon diversity index values for marmosets according to host environment and taxonomic classification, respectively (Figure 3A and 3B). Among Callithrix taxa, we see the highest median alpha diversity for the $C$. jacchus x $C$. penicillata hybrids and C. geoffroyi x C. penicillata hybrids. The median Shannon diversity index value is lowest for $C$. geoffroyi. Translocated and wild marmosets had higher median measure of Shannon diversity measures than captive marmosets. To better understand the effects of both host environment and taxon on marmoset alpha diversity, we fitted a series of ANOVA models (Table 2). We first fitted a model that included host environment and taxon as independent variables and Shannon index values as the dependent variable. A type I two-way ANOVA indicated that interaction between the independent variables is not significant. Under this model, host environment did have a significant effect on gut microbiome alpha diersity while host taxon did not. The Tukey post-hoc test indicated that differences in gut microbiome alpha diversity measures are significant between captive and translocated marmosets $(\mathrm{p}=0.0003)$. However, due to unbalanced design for both host environment and taxon, we then moved on to testing for effects of both independent variables on Callithrix gut microbiome diversity with a type II two-way ANOVA. Under this model, neither of the two independent variables were found to have a significant effect on the dependent variable (Table 2).

Table 2. ANOVA Models Fitted for Callithrix Gut Microbiome Alpha Diversity. 'DF' indicates degrees of freedom. 'F' indicates the F statistic.

\begin{tabular}{|c|c|c|c|c|c|c|c|c|c|c|}
\hline Model & Environment DF & Environment $\mathrm{F}$ & Environment p-value & Taxon DF & Taxon F & Taxon p-value & Interaction DF & Interaction $\mathrm{F}$ & Interaction Environment p-value & ANOVA Type \\
\hline Shannon Environment * Taxon & 2 & 8.59 & 0.0007 & 5 & 1.40 & 0.2426 & 3 & 0.33 & 0.80 & I \\
\hline Shannon ${ }^{2}$ Environment + Taxon & 2 & 0.31 & 0.7300 & 5 & 1.47 & 0.2187 & & & & II \\
\hline
\end{tabular}

We explored the effects of host taxon and environmental of marmoset gut microbiome beta diversity with the Bray-Curtis dissimilarity index. We plotted values of this measure on a non-metric multidimensional scaling (NMDS) ordination plot while superimposing both host environment and taxon onto the plot (Figure 3C). Visual inspection of the plot shows a stronger clustering pattern for host environment than for host taxon. We fit a PERMANOVA ${ }^{23}$ model with independent variable margins to explain the effects of host environment and taxon on marmoset gut microbiome Bray-Curtis indices. The effects of host environment were significant (PERMANOVA, $\mathrm{F}(2)=2.71, \mathrm{p}=0.001$ ) while those of host taxon were not (PERMANOVA, $\mathrm{F}(6)=1.25, \mathrm{p}=0.05)$. Post-hoc adonis pairwise tests ${ }^{24}$ of all possible combinations of host environmental levels were found to be significant ( $\mathrm{p}$-value $=0.001)$.

\section{Callithrix Gut Microbiome Bacterial Taxon Composition and Abundance}

As results for Callithrix gut microbiome diversity indicate a stronger effect on GMC of host environment than taxon, we focus this section on the effects of the former factor on bacterial abundance and composition in the Callithrix gut microbiome. Figure 4A shows relative abundances of bacterial classes for hosts according to their environmental classification. These plots shows that captive marmosets had relatively high abundance of Gammoproteobacteria (average abundance 61\%). Translocated marmosets seem to have relatively high abundance of Campylobacteria (average abundance 34\%) and Bacterioidia (18\%). Wild marmosets have an average relative abundance of Campylobacteria of 33\% and Actinobacteria average relative abundance of $41 \%$.

LefSe $^{25}$ differential gut microbiome bacteria abundance testing (Figure 4B and C) indicated significant differences between hosts when classifying them by environment. For host environment, LefSE analysis indicated in total 12 differentially abundant bacterial classes among marmoset hosts. Gammoproteobacteria, having the highest LDA score, was most enriched in captive marmosets. Actinobacteria was the class with the second highest LDA score and most abundant in wild marmosets. The bacterial class with the third highest LDA score was Campylobacteria, which was most abundant in translocated marmosets. At the bacterial genus level, LefSe analysis of host environment indicated that Bifidobacteria, which had the second highest LDA score, was most abundant in wild marmosets. The highest LDA bacterial genus could not be assigned for host environment. Helicobacter had the third highest LDA score and was most abundant in translocated marmosets.

\section{Diversity of Predicted Functional Pathways of the Callithrix Gut Microbiome}

PICRUST2 ${ }^{26}$ identified a total of 183 Kyoto Encyclopedia of Genes and Genomes (KEGG) predictive pathways among our sampled marmoset hosts. For alpha diversity of these KEGG pathways in the Callithrix gut, we first calculated separate Shannon diversity index values and grouped marmosets according to host environment and taxonomic classification, respectively (Figure 5A and 5B). Among Callithrix taxa, we see the highest median gut KEGG pathway alpha diversity for the $C$. jacchus $\mathrm{x} C$. penicillata hybrids and C. jacchus. The median Shannon diversity index value is lowest for C. geoffroyi $\mathrm{x} C$. penicillata hybrids. Translocated and captive marmosets show similar gut KEGG pathway median Shannon diversity measures, which were higher 
than that of wild marmosets. To better understand the effects of both host environment and taxon on gut KEGG pathway alpha diversity, we fitted ANOVA models (Table 3). We first fitted a model that included host environment and taxon as independent variables and Shannon index values as the dependent variable. A type I two-way ANOVA indicated that independent variable interaction is not significant. Under this model, host environment did have a significant effect on host gut KEGG pathway alpha diversity while host taxon did not (Table 3). However, due to the unbalanced design for both host environment and taxon, we then moved on to testing for effects of both independent variables on Callithrix gut KEGG pathway diversity with a type II two-way ANOVA. This ANOVA model indicated that host environment has a significant effect on host gut microbiome KEGG pathway alpha diversity (Table 3 ). The Tukey post-hoc test indicated that differences in alpha diversity measures are significant between wild and translocated marmosets $(\mathrm{p}=0.0000)$ as well as between wild and captive marmosets $(\mathrm{p}=0.0000)$.

Table 3. ANOVA Models Fitted for Callithrix Gut Predicted KEGG Pathways Alpha Diversity. 'DF' indicates degrees of freedom. ' $\mathrm{F}$ ' indicates the $\mathrm{F}$ statistics.

\begin{tabular}{|c|c|c|c|c|c|c|c|c|c|c|}
\hline Model & Environment DF & Environment $\mathrm{F}$ & Environment p-value & Taxon DF & Taxon F & Taxon p-value & Interaction DF & Interaction F & Interaction Environment p-value & ANOVA Type \\
\hline Shannon ${ }^{2}$ Environment * Taxon & 2 & 14.76 & 0.0000 & 6 & 0.35 & 0.9020 & 2 & \begin{tabular}{|l|}
0.76 \\
\end{tabular} & 0.4740 & I \\
\hline Shannon ${ }^{\sim}$ Environment + Taxon & 1 & 6.77 & 0.0137 & 3 & 0.28 & 0.8408 & & & & II \\
\hline
\end{tabular}

We plotted values of the Bray-Curtis dissimilarity index on a NMDS ordination plot while superimposing both host environment and taxon on to the plot (Figure 5C). Visual inspection of the plot shows a stronger clustering pattern for host environment than for host taxon. We fit a PERMANOVA model with independent variable margins to explain the effects of host environment and taxon on marmoset gut microbiome Bray-Curtis indices. Neither the effects of host environment (PERMANOVA, $\mathrm{F}(2)=1.85, \mathrm{p}=0.1390$ ) nor host taxon (PERMANOVA, $\mathrm{F}(6)=0.96, \mathrm{p}=0.493$ ) had a significant effect on Callithrix gut predicted KEGG pathway beta diversity.

\section{Callithrix Gut Microbiome KEGG Pathway Composition and Abundance}

For relative abundance of predicted KEGG pathways of the Callithrix gut microbiome, sampled marmoset distributions showed an even distribution of KEGG metabolism categories (Figure 6A). This pattern held regardless of host environmental categorization. Visual inspection of Figure 6A shows that the two most prominent metabolic KEGG metabolic categories were that of carbohydrate metabolism and amino acid metabolism. Figure 6B shows a heatmap of all predicted KEGG pathways across marmoset hosts, with accompanying host classification by environment and taxon, respectively. LEfSe analysis in Figure $6 \mathrm{C}$ shows significant enrichment of 15 predicted KEGG pathways in the marmoset gut, all specifically in captive hosts. The top enriched predicted KEGG gut microbiome pathway for captive marmosets was K0105, which had and LDA score of over 4.8 and is involved with carbohydrate metabolism of pectinesterase. The second most enriched predicted KEGG gut microbiome pathway for captive marmosets was K00660. This pathway had an LDA score of over 4.5 and is involved in flavinoid synthesis of chalcone synthase. The third most abundant KEGG pathway of K00072 was again most enriched in captive marmosets and had an LDA score of over 4.4. This pathway is involved with metabolism of co-factors and vitamins, specifically sepiapterin reductase.

BURRITO $^{27}$ linkage analysis between Callithrix gut bacterial taxa and predicted gut microbiome function are show in Figures 7 and Supplementary Figure 1 for major bacterial and functional classes. Visual inspection of the two figures shows overall that different sets of bacterial taxa are responsible for carrying out different functional roles between captive and wild marmosets. Two key differences in division of functional roles of gut bacterial taxa found in marmoset hosts in different environments is shown in Figure 7. Figure 7A show that Actinobacteria take on a number of functional roles in the Callithrix gut microbiome almost exclusively within wild hosts. When Actinobacteria are expanded to the genus level and metabolism is expanded to lower pathway levels, Bifidobacterium seems especially important among wild marmosets for carbohydrate and amino acid metabolism (Figure 7B). On the other hand, Proteobacteria seem to be heavily involved across variable major functions in the gut of captive and translocated marmosets (Figure 7C and D). When Proteobacteria are expanded to the family level, Enterobacteriaceae seem to be carrying out a large number of functional roles, including across major categories of metabolic pathways in captive and translocated marmosets (Figure 7D). For other classes of bacteria found in the Callithrix gut, Bacteroidota, Firmicutes, and Campilobacterota seem to take on a broad number of functional roles in both translocated and captive marmosets (Supplementary Figure 1B and C). The latter two also seems to also perform broad gut functional roles in a smaller subset of captive marmoset hosts (Supplementary Figure 1C).

\section{Discussion}

The individual gut microbiomes of marmosets sampled in this study possessed similar classes of bacterial phyla such as Bacteroidia, Actinobacteria (especially Bifidobacteria), Campylobacteria, and Gammaproteobacteria. This result may be linked to how closely related marmoset species are ${ }^{16}$. However, the abundance of marmoset gut bacterial phyla differed starkly 
between hosts sampled in different environments. In fact, our results reveal that host environmental factors significantly shape the composition and function of the gut microbiome. Therefore, our discussion on the results of this study largely focus on how such factors may influence the Callithrix GMC.

Several studies already suggest that Bifidobacterium is a key component of the Callithrix gut microbiome to support carbohydrate metabolism. Across primates, the genus Callithrix along with the closely related Leontopithecus are the two primate genera with the highest average abundance of Bifidobacterium $(>30 \%)$ in the primate gut microbiome, followed by members of the Hominidae family $(10 \%)^{28}$. While Lugili et al. $(2020)^{28}$ did not use the same marmoset species as our study, they did observed evolutionary associations between specific Bifidobacterium species and specific callitrichid hosts. The gut microbiome of callitrichids had high average abundance of the B. tissieri group ${ }^{28}$. This Bifidobacteria group is especially efficient at metabolizing carbon sources like arabinogalactan and pectin ${ }^{28}$, which are components of carbohydrates of plant gums consumed by marmosets ${ }^{29}$. The genomes of three isolates of Bifidobacterium callitrichos from a captive C. jacchus fecal sample contained predicted genes associated with galactose and arabinose metabolism, which are also major constituents of tree gums eaten by $C$. jacchus ${ }^{30}$. In 3 US captive facilities, $C$. jacchus collectively shared four species of Bifidobacterium, which possessed genes encoding ATP-binding cassette proteins important for nutrient transport that may be specific to the marmoset gut ${ }^{31}$.

In our sample, the gut microbiome of wild Callithrix was significantly enriched for the bacterial class of Actinobacteria, especially Bifidobacterium. This bacterial genus was also observable in the gut microbiome of captive and translocated marmosets but to a much lesser degree. While our sample size of wild marmosets was smaller relative to the number of captive and translocated/marmosets, our results are nonetheless consistent with other studies that show that Bifidobacteria are a key component of the marmoset gut microbiome. Our data are the first to show that Bifidobacteria seem to be an integral part of the wild Callithrix gut microbiome. However, we were not able to determine the exact species of Bifidobacteria present in the gut of wild marmosets. Thus, an important next step in marmoset microbiome studies will be to expand study of wild marmosets and resolve wild Callithrix GMC at the bacterial species level.

In contrast to wild marmosets, the captive marmoset gut microbiome was overwhelming enriched for the Gammaproteobacteria bacterial class, and in particular from the family Enterobacteriaceae. The GMC of marmosets in captivity shows similarity to certain aspects of the GMC of human GI diseases associated with gut microbiome dysbiosis ${ }^{4,13,18,32}$. Crohn's Disease (CD) generally affects the terminal ileum and colon and GMC of CD sufferers is less diverse relative to healthy humans ${ }^{13,33}$. The gut microbiome of CD patients is enriched for bacterial taxa that include Enterobacteriaceae and depleted for Bifidobacteriaceae ${ }^{13,34}$, as we similarly observed in non-wild marmosets. A similar pattern was observed in captive $C$. jacchus clinically diagnosed with intestinal bowel disease ${ }^{18}$. Enterobacteriaceae is frequently associated with intestinal diseases and contains a number of pathogenic bacterial strains of Salmonella, Escherichia, and Shigella ${ }^{35,36}$. Perhaps the presence of Enterobacteriaceae in healthy captive marmosets makes them more susceptible for developing eventual GI problems, as this is a shift away from the eubiosis of natural marmoset GMC.

Translocated marmoset GMC shows similarity to that of captive marmoset in being significantly enriched for the Proteobacteria phylum. However, translocated hosts possess a greater diversity of bacteria taxa within this phylum, as opposed to the higher gut Enterobacteriaceae abundance in captive hosts. One enriched Proteobacteria species of note in translocated Callithrix guts was Helicobacter, of which certain species like H. pylori are known to cause gastric disease in humans ${ }^{37}$. Another Proteobacteria species which was enriched in translocated marmoset guts was Campylobacter. This bacterial genus is associated with diarrhea illness in humans ${ }^{38}$. Bacteroidetes and Clostridia were also significantly abundance in the gut of translocated marmosets, a pattern also seen in the human GI disease of ulcerative colitis ${ }^{34}$.

In a survey of gut microbiota of healthy $C$. jacchus at captive institutions, the most abundant phylya were Actinobacteria, Bacterioidete, Firmicutes, Fusobacteria, and Proteobacterioa ${ }^{18}$. From these institutions, most reported the greatest average abundance of Bacteroidetes, followed by three captive facilities with highest average abundance of Proteobacteria, and then three other institutions which reported Actinobacteria have the highest gut abundance. Therefore, it seems that at least in captivity, $C$. jacchus does not have a single GMC signature. Marmoset gut bacterial composition and abundance vary between biomedical institutions found outside of Brazil. In our case, marmosets sampled in captivity within Brazil overwhelmingly showed a high abundance of Gammaproteobacteria. On the other hand, studies of the gut microbiome of $C$. jacchus with GI disease show various changes such as Bifidobacteria abundance, rise in Clostridium sensu stricto, and the presence of Enterobacteriaceae in the cases of marmosets with inflammatory bowel disease ${ }^{18}$.

Despite the differences in gut bacterial composition and abundance among the marmosets in our sample, the gut microbiome of our sampled marmosets seems to perform the same set of broad functions. Carbohydrate and amino acid metabolism are among the major functions carried out by the Callithrix gut microbiome in this study. However, closer inspection shows differential abundance of specific KEGG pathways between marmoset hosts from different environments. Further, there seems to be a stark difference in the distribution of functional roles among bacterial taxa found in the Callithrix gut microbiome in captive, translocated, and wild marmosets. This study is one of the first to show linkage between gut microbiome function 
and bacterial taxa. Conversely, a relatively wider diversity of bacterial taxa take on functional roles in the gut microbiome of translocated and wild marmosets. Bifidobacteria seems to take a prominent role in amino acid and carbohydrate metabolism in wild marmosets, a pattern not replicated in non-wild marmosets. Instead, in captive and translocated marmosets, Proteobacteria seem to dominate functional roles of the gut microbiome. In captive marmosets, Enterobacteriaceae seem to dominate all aspects of gut microbiome function.

Based on the above data above, one likely important environmental factor to consider in its influence on Callithrix GMC is diet. Wild Callithrix regularly exploit tree gums or hardened saps ${ }^{14,15}$, and marmoset species are among only 27 mammals classified as obligate exudivores ${ }^{13}$. The chemical composition of sugars in the marmoset diet have be most explored in Callithrix jacchus, and include beta-linked polyssachrides composed on galactose, arabinose, and rhamnose ${ }^{30}$. Additionally, pectin is another carbohydrate found in the bark of Anadenanthera peregrina, which is consumed by various taxa of marmosets ${ }^{29}$. Further, marmosets morphological adaptations for exudivory include a well-developed caecum for fermentation of plant exudates $^{39}$ and a lower-anterior dentition "tooth scraper" to perforate tree bark and lianas ${ }^{40}$. Additionally, wild marmosets also generally exploit other nutritional sources such as fruit, fungi, and small prey ${ }^{16,41}$. In captivity, marmosets diets do not reflect what marmosets would normally eat in nature. Most captive institutions do not supplement marmoset diets with gum, and instead they generally combine different proportions of commercial chow, fruits, vegetables, protein, and sweets ${ }^{42}$. When gum is supplied to marmosets in captivity, the most commonly used source is arabica gum ${ }^{42}$. Captive Brazilian facilities from where we sampled marmosets for this study also follow similar husbandry practices for marmoset nutrition (Table 4) as that described by Goodroe et al $(2021)^{42}$.

Table 4. Diet collectively fed to marmoset hosts in sampled captive facilities.

\begin{tabular}{|l|l|}
\hline Fruits & Papaya, Orange, Banana, Apple, Pear, Avacado, Kiwi, Melon, Mango \\
\hline Carbs & Sweet Potato, Potato, Beets \\
\hline Vegetables & Cucumber, Eggplant, Pumpkin, Chuchu, Cauliflower, Carrots \\
\hline Proteins & Cooked Chicken, Cooked Egg \\
\hline
\end{tabular}

Thus, one concern for a lack of tree gums in the diet of captive specialist exudivores is the development of health issues as well as a negative impact on breeding and survivability ${ }^{43,44}$. Indeed, institutions with captive marmosets report GI disease such as inflammatory-like bowel disease, chronic malabsorption, and chronic diarrhea among the most common clinical syndromes of captive marmosets (which are largely $C$. jacchus) ${ }^{42}$. In humans suffering from GI diseases, increasing plant-based foods and dietary fiber, resulted in increasing microbiome diversity, remission of GI symptoms, and decreasing risk of GI ${ }^{4,45}$. Such diets may increase gut abundance of bacteria such as Bifibacterium that produce short chain fatty acids like butyrate, which may guard against proliferation of pathogenic bacteria in the gut and decrease chronic inflammation ${ }^{4,45}$.

Animal studies increasingly recognize the need to include host-microbiome associations in management of captive populations ${ }^{2,12}$. It has also been demonstrated, as in mice and wood-rats, that feeding more natural diets to individuals in captivity helped maintain host GMC profiles closer to wild, free-ranging hosts ${ }^{46,47}$. Lack of access to a natural-wild diet for marmosets and other exudivory specialists may also promote loss of native gut microbes like Bifidobacterium and enrichment of potentially pathogenic bacterial strains of Enterobacteriaceae. Additionally, high abundance of Bifidobacterium in the gut microbiomes may be a key biomarker for host gut microbiome eubiosis in marmosets ${ }^{18}$ and other mammalian exudivores. Thus, based on such observations, captive facilities should consider provisioning of gum in the diet of specialized exudivores to improve host welfare by maintaining gut microbiomes closer to that of wild populations.

\section{Conclusion}

Our major findings indicate that, just as in other primates, the gut microbiome of Callithrix is sensitive to environmental factors, and that carbohydrate metabolism is a key function of the Callithrix gut microbiome. Yet, although marmosets are specialized exudivores, there is a wide gap between the nutritional intake of marmosets in the wild and captivity. From an evolutionary and health point of view, this disconnect between wild and captive Callithrix diets leaves open several questions concerning maladaptation of the marmoset digestive system, perturbation of gut microbiota, and long term health effects. More studies are needed to understand better the health and reproductive consequences of omitting as well as increasing gum intake by specialized exudivores in captivity. Also, systematic studies are needed to better understand whether Bifidobacterium plays a central role in maintaining euobisis in the gut microbiomes of Callithrix and other exudivory specialists. Overall, such information will expand baseline gut microbiome data available for wild and non-wild exudivores to allow for the development of new tools to improve exudiviore management, welfare, and conservation. 


\section{Methods}

\section{Sample Collection}

We collected anal swabs between 2015 and 2016 from 59 adult individuals (Figure 1, Table 1 and Supplementary Table 1). Marmoset sampling was authorized by the Brazilian Environmental Ministry (SISBIO protocol\# 47964-2), the Arizona State University IACUC (protocol\# 15-144R). Wild animals were captured with Tomahawk style traps baited with bananas. Sampled animals were immobilized by ketamine $(10 \mathrm{mg} / \mathrm{kg}$ of body weight) inner thigh intramuscular injection, photographed, weighed, measured, examined clinically by veterinarians, and deemed healthy upon examination. Copan FLOQ Swabs were gently rotated in the anal region and submerged in storage buffer $(50 \mathrm{mM}$ Tris $\mathrm{pH} 8.0,50 \mathrm{mM}$ EDTA, $50 \mathrm{mM} \mathrm{Sucrose,} 100 \mathrm{mM} \mathrm{NaCl}$, $1 \%$ SDS) before being discarded. After processing, animals were returned to cages for recovery. Wild marmosets were released at original capture sites.

\section{Sample Processing and Sequencing}

Bacterial DNA extraction from Callithrix anal swabs was carried out by following a modified phenol-chloroform protocol ${ }^{48}$. Modifications included beating the samples on a vortex fitted with a horizontal vortex adaptor (\#13000-V1-24, Mo Bio, Carlsbad, CA, USA) for 10 minutes at step "2Aiii," precipitating samples in $100 \%$ ethanol in step "2Axvi" and rehydrating DNA pellets in 25 uL low TE buffer at step "2Axxii." Extracted DNA was quantified on a Qubit3 (Life Technologies, Carlsbad, CA, USA) with a dsDNA HS Assay Kit (Life Technologies). DNA samples obtained for this study have been registered in the Brazilian SISGen database under entries \# A2E885E, A965629, A5CB6FA, AE784B5, and A07A291. The V4 region of the bacterial 16S rRNA gene was amplified from sampled DNA in triplicate using the barcoded primer set 515f/806 ${ }^{49}$.

Amplicon triplicates were combined for each individual and then pooled in equimolar amounts into a multiplexed Illumina sequencing library. The library was purified with a Zymo DNA Concentrator and Cleaner-5 (\#D4013, Zymo Research, Irving, CA, USA) and size selected for 375-380 base pairs with Agencourt Ampure XP (\#A63880, Beckman Coulter, Indianapolis, IN, USA) magnetic beads. Libraries were sequenced at Arizona State University, USA on an Illumina MiSeq for2x250 cycles.

\section{Bioinformatics and Statistical Analysis}

Code for bioinformatics analysis described below is available at github.com/Callithrix-omics/callithrix_ microbiome. Data were demultiplexed using default parameters in Qiime2-2021.2 $2^{21}$ The DADA2 Qiime2 plug-in ${ }^{50}$ was used to quality-filter and trim sequences and join paired-end reads. Upon trimming, the first 10 and last 30 nucleotides were removed from reverse reads due to low base quality. These steps resulted in feature tables of DNA sequences and their per-sample counts. MAAFT ${ }^{51}$ and FastTree ${ }^{52}$, as part of the Qiime2 phylogeny plug-in aligned and produced a midpointed rooted phylogenetic tree of feature sequences. Taxonomic composition of samples was determined with the Qiime2 Naive Bayes q2-feature-classifier plug-in, which was trained on pre-formatted SILVA reference sequence and taxonomy files "Silva 138 SSURef NR99 515F/806R region sequences" and "Silva 138 SSURef NR99 515F/806R region taxonomy"53-55 for the portion of the $16 \mathrm{~S} \mathrm{V4}$ region bounded by the $515 \mathrm{~F} / 806 \mathrm{R}$ primer pair. The pre-formated files were downloaded from docs.qiime2.org/2021.4/data-resources. Taxonomic classification of the feature table was carried out with the q2-feature-classifier classify-sklearn command. For further down stream analyses, we used the Qiime2 export option to extract a biom format file from the classified feature table as well as feature table taxonomic information. Information from the exported biom file and feature table taxonomy were merged into a new biom format file with the biom $2.1 .10^{56}$ command line tool.

For community profiling and comparative analysis, we used the Marker-gene Data Profiling (MDP) module of the MicrobiomeAnalyst web-based platform ${ }^{22}$, using the merged biom file from above as well as sample metadata given in Supplementary Table 1. At the MicrobiomeAnalyst data filtering step, we let the default settings of the 'Low count filter' to a minimum count of 4 and $20 \%$ prevalence in samples and the 'percentage to remove' option under 'Low variance filter' set to $10 \%$ based on the interquantile range. Next, at the data normalization step, we chose to rarefy the data to the minimum library size, data was scaled by 'total sum scaling,' and we did not apply any data transformations.

Alpha-diversity measures were calculated for normalized and filtered data in in MicrobiomeAnalyst with settings configured for host species and environment, respectively, taxonomic level as feature, Shannon index as the diversity measure, and statistical significance as T-test/ANOVA. To better understand the effects of both host environment and taxon on marmoset alpha diversity, we fitted a series of ANOVA models to test for the effects of host taxon and environment on Callithrix gut microbiome alpha diversity. We carried out this analysis in $\mathrm{R}$ because it allows for much finer grain control over statistical tests than MicrobiomeAnalyst, thus we exported individual host Shannon diversity index measures from the program. We first tested a two-way Type I ANOVA model that included host environment and taxon as independent variables and the interaction between them. We then fit a two-way Type II ANOVA with host environment and taxon as independent variable to account of the unbalanced design for both host factors for our samples. The Type II ANOVA was fit with the R car ${ }^{57}$ package. Diagnostic Q-Q and Residual vs Fitted plots were made for the final model indicated an appropriate fit of our model to the data. 
To explore beta diversity of the Callithrix gut microbiome, we calculated the Bray-Curtis dissimilarity indices for each host, and then used the indices to make a NMDS ordination plot in the R vegan program ${ }^{58}$. We superimposed both environmental and taxon information for each marmoset on to the NMDS plot. To understand the effects of host environment and taxon had an effect on marmoset gut microbiome Bray-Curtis dissimilarity indices, we used adonis 2 function in the phyloseq package ${ }^{23}$. We fitted PERMANOVA ${ }^{51}$ models which included the marginal effects of host environment and taxon as independent variables and Bray- Curtis dissimilarity indices as the dependent variable. Simulation studies have found that PERMANOVA is robust to unbalanced sampling designs ${ }^{59}$. The PERMANOVA model was ran with the adnois 2 function. PERMANOVA post-hoc tests of the Bray-Curtise dissimilarity indices were carried out as pairwise adonis tests with the adonis.pair function from the the EcolUtils ${ }^{24} \mathrm{R}$ package. The test was run for 1000 permutations and p-values were corrected by the false discovery rate.

To profile bacterial taxa abundance, we used MicrobiomeAnalyst to generate stacked bars of relative bacterial abundance at the class level for hosts according to host taxon and captivity, respectively. Taxa resolution settings were set to merge small taxa with total counts of less than 10 . To test for significance in differential bacterial taxa abundance according to host environment and taxon, respectively, we applied two different approaches. First, we used LEfSe ${ }^{25}$ at the genus level for bacterial taxa within MicrobiomeAnalyst with the default settings of a FDR-adjusted p-value cutoff set to 0.1 and the log LDA cut-off at 2.0. We applied LEFse separately for host environmental and taxon classifications.

To explore the functional aspects of the Callithrix gut microbiome, the Kyoto Encyclopedia of Genes and Genome Orthology (KO) pathways were predicted with PICRUSt ${ }^{26}$ by following guidelines at https: / / github. com/picrust/ picrust 2 /wiki. First, predicted KEGG ORTHOLOGY (KO) functional predictions were carried out with the

metagenome_pipeline.py script with the -strat_out option. By default, PICRUSt2 excluded all features with the nearest sequenced taxon index (NSTI) value $>2$ from the output. The average weighted NSTI value of the data set after this automatic filtering was $0.08 \pm 0.12 \mathrm{SD}$. Then Kyoto Encyclopedia of Genes and Genomes (KEGG) pathway abundances were derived from predicted KO abundances were performed with the "-no_regroup" option in the pathway_pipeline.py script in PICRUST2.

We then rounded the unstratified KEGG pathway abundance results for alpha and beta analysis of predicted functional pathways of the Callithrix gut microbiome. We then turned these results into a phyloseq object in R. We first used phyloseq to estimate the Shannon diversity index for Callithrix gut microbiome predicted KEGG pathways and then fit ANOVA models to test the effects of host environment and taxon on these index values. We first tested a two-way Type I ANOVA model that included host environment and taxon as independent variables and the interaction between them. We then fit a two-way Type II ANOVA with host environment and taxon as independent variable to account of the unbalanced design for both independent variables. The Type II ANOVA was fit with the R car ${ }^{57}$ package. Diagnostic Q-Q and Residual vs Fitted plots for this final model indicated an appropriate fit of our model to the data. Post-hoc analyses for this model were performed with Tukey's HSD test.

Using PICRUST2 unstratified KEGG pathway abundance results, we generated a relative abundance plot of Callithrix gut microbiome KEGG metabolic processes using the Shotgun Data Profiling Module in MicrobiomeAnalyst. At the MicrobiomeAnalyst data filtering step, we let the default settings of the 'Low count filter' to a minimum count of 4 and $20 \%$ prevalence in samples and the 'percentage to remove' option under 'Low variance filter' set to $10 \%$ based on the interquantile range. Data After MicrobiomAnalyst filters, a total of $137 \mathrm{KEGG}$ pathways remained for further analysis. A functional diversity relative abundance plot was generated for KEGG metabolism based on category abundance total hits. As KEGG pathway diversity analysis indicated a stronger effect of host environment, we grouped this abundance plot by this host factor. We tested for significant patterns of differnetial abundance between host environment categories in MicrobiomeAnalyst uing LEfSe using a FDR-adjusted p-value cutoff of 0.1 and Log LDA score of 2.0.

BURRITO $^{27}$, an online interactive visualization module, was used to make links between our bacterial abundance data and predicted functional profiles from the Callithrix gut microbiome. As input, we used taxonomic abundance and bacterial taxonomy data based on the biom file originally extracted from Qiime 2. We also provided a function attribution table based on PICRUST2 output that linked the functional and taxonomic data by following instructions for the convert_table.py script at https://github.com/picrust/picrust2/wiki. We also provided a metadata table to BURRITO which included host environmental classifications.

\section{References}

1. Huttenhower, C. et al. Structure, function and diversity of the healthy human microbiome. Nature 486, 207-214, DOI: 10.1038/nature11234 (2012).

2. West, A. G. et al. The microbiome in threatened species conservation. Biol. Conserv. 229, 85-98, DOI: 10.1016/j.biocon. 2018.11.016 (2019).

3. Cho, I. \& Blaser, M. J. The human microbiome: at the interface of health and disease. Nat. Rev. Genet. 13, 260-270, DOI: $10.1038 / \mathrm{nrg} 3182$ (2012). 
4. DeGruttola, A. K., Low, D., Mizoguchi, A. \& Mizoguchi, E. Current understanding of dysbiosis in disease in human and animal models. Inflamm. Bowel Dis. 22, 1137-1150, DOI: 10.1097/mib.0000000000000750 (2016).

5. Clayton, J. B. et al. Captivity humanizes the primate microbiome. Proc. Natl. Acad. Sci. 113, 10376-10381, DOI: 10.1073/pnas.1521835113 (2016).

6. Ley, R. E. et al. Evolution of mammals and their gut microbes. Science 320, 1647-1651, DOI: 10.1126/science.1155725 (2008).

7. Youngblut, N. D. et al. Host diet and evolutionary history explain different aspects of gut microbiome diversity among vertebrate clades. Nat. Commun. 10, DOI: 10.1038/s41467-019-10191-3 (2019).

8. McKenzie, V. J. et al. The effects of captivity on the mammalian gut microbiome. Integr. Comp. Biol. 57, 690-704, DOI: 10.1093/icb/icx090 (2017).

9. Houtz, J. L., Sanders, J. G., Denice, A. \& Moeller, A. H. Predictable and host-species specific humanization of the gut microbiota in captive primates. Mol. Ecol. DOI: 10.1111/mec.15994 (2021).

10. Frankel, J. S., Mallott, E. K., Hopper, L. M., Ross, S. R. \& Amato, K. R. The effect of captivity on the primate gut microbiome varies with host dietary niche. Am. J. Primatol. 81, DOI: 10.1002/ajp.23061 (2019).

11. Gevers, D. et al. The treatment-naive microbiome in new-onset crohn's disease. Cell Host \& Microbe 15, 382-392, DOI: 10.1016/j.chom.2014.02.005 (2014).

12. Pineda-Munoz, S. \& Alroy, J. Dietary characterization of terrestrial mammals. Proc. Royal Soc. B: Biol. Sci. 281, 20141173, DOI: 10.1098/rspb.2014.1173 (2014).

13. CABANA, F., DIERENFELD, E. S., Wirdateti, DONATI, G. \& NEKARIS, K. A. I. Exploiting a readily available but hard to digest resource: A review of exudativorous mammals identified thus far and how they cope in captivity. Integr. Zool. 13, 94-111, DOI: 10.1111/1749-4877.12264 (2018).

14. Nash, L. T. Dietary, behavioral, and morphological aspects of gummivory in primates. Am. J. Phys. Anthropol. 29, 113-137, DOI: 10.1002/ajpa.1330290505 (1986).

15. Smith, A. C. Exudativory in primates: Interspecific patterns. In The Evolution of Exudativory in Primates, 45-87, DOI: 10.1007/978-1-4419-6661-2_3 (Springer New York, 2010).

16. Malukiewicz, J. et al. An introduction to the callithrix genus and overview of recent advances in marmoset research. Preprints DOI: 10.20944/preprints202011.0256.v2 (2021).

17. Ludlage, E. \& Mansfield, K. Clinical care and diseases of the common marmoset (callithrix jacchus)). Comp. Medicine 53, 369-382 (2003).

18. Sheh, A. The gastrointestinal microbiota of the common marmoset (callithrix jacchus). ILAR J. DOI: 10.1093/ilar/ilaa025 (2021).

19. Hershkovitz, P. Living new world monkeys (Platyrrhini): With an introduction to primates. (University of Chicago Press, Chicago, IL, 1977).

20. Fuzessy, L. F. et al. Morphological variation in wild marmosets (callithrix penicillata and c. geoffroyi) and their hybrids. Evol. Biol. 41, 480-493, DOI: 10.1007/s11692-014-9284-5 (2014).

21. Bolyen, E. et al. Reproducible, interactive, scalable and extensible microbiome data science using QIIME 2 . Nat. Biotechnol. 37, 852-857, DOI: 10.1038/s41587-019-0209-9 (2019).

22. Chong, J., Liu, P., Zhou, G. \& Xia, J. Using MicrobiomeAnalyst for comprehensive statistical, functional, and meta-analysis of microbiome data. Nat. Protoc. 15, 799-821, DOI: 10.1038/s41596-019-0264-1 (2020).

23. McMurdie, P. J. \& Holmes, S. phyloseq: An r package for reproducible interactive analysis and graphics of microbiome census data. PLoS ONE 8, e61217, DOI: 10.1371/journal.pone.0061217 (2013).

24. Salazar, G. EcolUtils: Utilities for community ecology analysis (2021). R package version 0.1 .

25. Segata, N. et al. Metagenomic biomarker discovery and explanation. Genome Biol. 12, R60, DOI: 10.1186/ gb-2011-12-6-r60 (2011).

26. Douglas, G. M. et al. PICRUSt 2 for prediction of metagenome functions. Nat. Biotechnol. 38, 685-688, DOI: 10.1038/ s41587-020-0548-6 (2020).

27. McNally, C. P., Eng, A., Noecker, C., Gagne-Maynard, W. C. \& Borenstein, E. BURRITO: An interactive multi-omic tool for visualizing taxa-function relationships in microbiome data. Front. Microbiol. 9, DOI: 10.3389/fmicb.2018.00365 (2018). 
28. Lugli, G. A. et al. Evolutionary development and co-phylogeny of primate-associated bifidobacteria. Environ. Microbiol. 22, 3375-3393, DOI: 10.1111/1462-2920.15108 (2020).

29. Francisco, T. M. et al. Feeding habits of marmosets: A case study of bark anatomy and chemical composition ofAnadenanthera peregrinagum. Am. J. Primatol. 79, e22615, DOI: 10.1002/ajp.22615 (2016).

30. Albert, K., Rani, A. \& Sela, D. A. The comparative genomics of bifidobacterium callitrichos reflects dietary carbohydrate utilization within the common marmoset gut. Microb. Genomics 4, DOI: 10.1099/mgen.0.000183 (2018).

31. Brown, C. J. et al. Comparative genomics of bifidobacterium species isolated from marmosets and humans. Am. J. Primatol. 81, DOI: 10.1002/ajp.22983 (2019).

32. Ott, S. J. Reduction in diversity of the colonic mucosa associated bacterial microflora in patients with active inflammatory bowel disease. Gut 53, 685-693, DOI: 10.1136/gut.2003.025403 (2004).

33. Pérez-Brocal, V. et al. Metagenomic analysis of crohn's disease patients identifies changes in the virome and microbiome related to disease status and therapy, and detects potential interactions and biomarkers. Inflamm. Bowel Dis. 21, 2515-2532, DOI: 10.1097/mib.0000000000000549 (2015).

34. Alam, M. T. et al. Microbial imbalance in inflammatory bowel disease patients at different taxonomic levels. Gut Pathog. 12, DOI: 10.1186/s13099-019-0341-6 (2020).

35. Paterson, D. L. Resistance in gram-negative bacteria: Enterobacteriaceae. The Am. J. Medicine 119, S20-S28, DOI: 10.1016/j.amjmed.2006.03.013 (2006).

36. Kang, E. et al. Enterobacteria and host resistance to infection. Mammalian Genome 29, 558-576, DOI: 10.1007/ s00335-018-9749-4 (2018).

37. Camilo, V., Sugiyama, T. \& Touati, E. Pathogenesis ofHelicobacter pyloriinfection. Helicobacter 22, e12405, DOI: 10.1111/hel.12405 (2017).

38. Fitzgerald, C. Campylobacter. Clin. Lab. Medicine 35, 289-298, DOI: 10.1016/j.cll.2015.03.001 (2015).

39. Power, M. L. \& Myers, E. W. Digestion in the common marmoset (callithrix jacchus), a gummivore-frugivore. Am. J. Primatol. 71, 957-963, DOI: 10.1002/ajp.20737 (2009).

40. Coimbra-Filho, A. \& Mittermeier, R. Tree-gouging, exudate-eating, and the 'short-tusked' condition in callithrix and cebuella. In DG, D. K. (ed.) The biology and conservation of the Callitrichidae., 105-15 (Washington, DC: Smithsonian Institution Press, 1976).

41. Martins, M. \& Setz, E. Diet of buffy tufted-eared marmosets (callithrix aurita) in a forest fragment in southeastern brazil. Int. J. Primatol. 21, 467-476 (2000).

42. Goodroe, A. et al. Current practices in nutrition management and disease incidence of common marmosets ( callithrix jacchus ). J. Med. Primatol. 50, 164-175, DOI: 10.1111/jmp.12525 (2021).

43. Cabana, F. \& Nekaris, K. Diets high in fruits and low in gum exudates promote the occurrence and development of dental disease in pygmy slow loris (nycticebus pygmaeus). Zoo Biol. 34, 547-553, DOI: 10.1002/zoo.21245 (2015).

44. Cabana, F., Maguire, R., Hsu, C.-D. \& Plowman, A. Identification of possible nutritional and stress risk factors in the development of marmoset wasting syndrome. Zoo Biol. 37, 98-106, DOI: 10.1002/zoo.21398 (2018).

45. Grosse, C. S. J., Christophersen, C. T., Devine, A. \& Lawrance, I. C. The role of a plant-based diet in the pathogenesis, etiology and management of the inflammatory bowel diseases. Expert. Rev. Gastroenterol. \& Hepatol. 14, 137-145, DOI: 10.1080/17474124.2020.1733413 (2020).

46. Martínez-Mota, R., Kohl, K. D., Orr, T. J. \& Dearing, M. D. Natural diets promote retention of the native gut microbiota in captive rodents. The ISME J. 14, 67-78, DOI: 10.1038/s41396-019-0497-6 (2019).

47. Leeuwen, P., Mykytczuk, N., Mastromonaco, G. F. \& Schulte-Hostedde, A. I. Effects of captivity, diet, and relocation on the gut bacterial communities of white-footed mice. Ecol. Evol. 10, 4677-4690, DOI: 10.1002/ece3.6221 (2020).

48. Sambrook, J. \& Russel, D. Molecular cloning (Cold Spring Harbor: CSHL Press, 2001).

49. Caporaso, J. G. et al. Ultra-high-throughput microbial community analysis on the illumina HiSeq and MiSeq platforms. The ISME J. 6, 1621-1624, DOI: 10.1038/ismej.2012.8 (2012).

50. Callahan, B. J. et al. DADA2: High-resolution sample inference from illumina amplicon data. Nat. Methods 13, 581-583, DOI: $10.1038 /$ nmeth.3869 (2016). 
51. McMurdie, P. J. \& Holmes, S. phyloseq: An $\mathrm{r}$ package for reproducible interactive analysis and graphics of microbiome census data. PLOS ONE 8, 1-11, DOI: 10.1371/journal.pone.0061217 (2013).

52. Price, M. N., Dehal, P. S. \& Arkin, A. P. FastTree 2 - approximately maximum-likelihood trees for large alignments. PLoS ONE 5, e9490, DOI: 10.1371/journal.pone.0009490 (2010).

53. Quast, C. et al. The SILVA ribosomal RNA gene database project: improved data processing and web-based tools. Nucleic Acids Res. 41, D590-D596, DOI: 10.1093/nar/gks1219 (2012).

54. Yilmaz, P. et al. The SILVA and "all-species living tree project (LTP)" taxonomic frameworks. Nucleic Acids Res. 42, D643-D648, DOI: 10.1093/nar/gkt1209 (2013).

55. Glöckner, F. O. et al. 25 years of serving the community with ribosomal RNA gene reference databases and tools. $J$. Biotechnol. 261, 169-176, DOI: 10.1016/j.jbiotec.2017.06.1198 (2017).

56. McDonald, D. et al. The biological observation matrix (BIOM) format or: how i learned to stop worrying and love the ome-ome. GigaScience 1, DOI: 10.1186/2047-217x-1-7 (2012).

57. Fox, J. \& Weisberg, S. An R Companion to Applied Regression (Sage, Thousand Oaks CA, 2019), third edn.

58. Oksanen, J. et al. vegan: Community Ecology Package (2020). R package version 2.5-7.

59. Anderson, M. J. \& Walsh, D. C. I. PERMANOVA, ANOSIM, and the mantel test in the face of heterogeneous dispersions: What null hypothesis are you testing? Ecol. Monogr. 83, 557-574, DOI: 10.1890/12-2010.1 (2013).

\section{Acknowledgements}

We thank Vanner Boere, Ita de Oliveira, Rodrigo S. Carvalho, the Guarulhos Zoo, the CEMAFAUNA, the CPRJ staff, SERCAS staff, and AMLD staff for assistance with wild and captive populations. We are very grateful to Cecil M. Lewis and Tanvi Honap at LMAMR for donation of PCR primers. We thank Dietmar Zinner, Corinna Ross, and four anonymous reviewers for comments on this work.

\section{Author contributions statement}

JM and ADG formulated the idea for the study. JM collected samples, obtained funding, conducted wet and dry laboratory work, and wrote the original manuscript. RAC provided study guidance, and logistical support. JAD, SK, LTN, ATO provided study guidance, and logistical support. CSI gave access and provided logistical support to collect samples from animals kept at Guarulhos Zoo. SBM provided logistical support and veterinary assistance to collect samples from animals kept at the Rio de Janeiro Primatology Center. PAN gave access and provided logistical support to collect samples from animals kept at CEMAFAUNA. AAQ provided logistical support and veterinary assistance to collect samples from animals kept at CEMAFAUNA. LCMP gave access and provided logistical support to collect samples from animals kept at CEMAFAUNA. AP gave access and provided logistical support to collect samples from animals kept at CPRJ. CRRM was a major contributors in writing the manuscript and provided access to collect samples from SERCAS marmosets. DLS performed DNA extractions, methodological optimization, and carried out PCR. ACS was a major contributor in writing the manuscript, provided study guidance, and logistical support. CR gave significant guidance and input in the development of this study. All authors read and approved the final manuscript.

\section{Additional information}

The dataset supporting the conclusions of this article is available in the NCBI SRA repository under Bioproject PRJNA574641. The authors declare that they have no competing interests. 


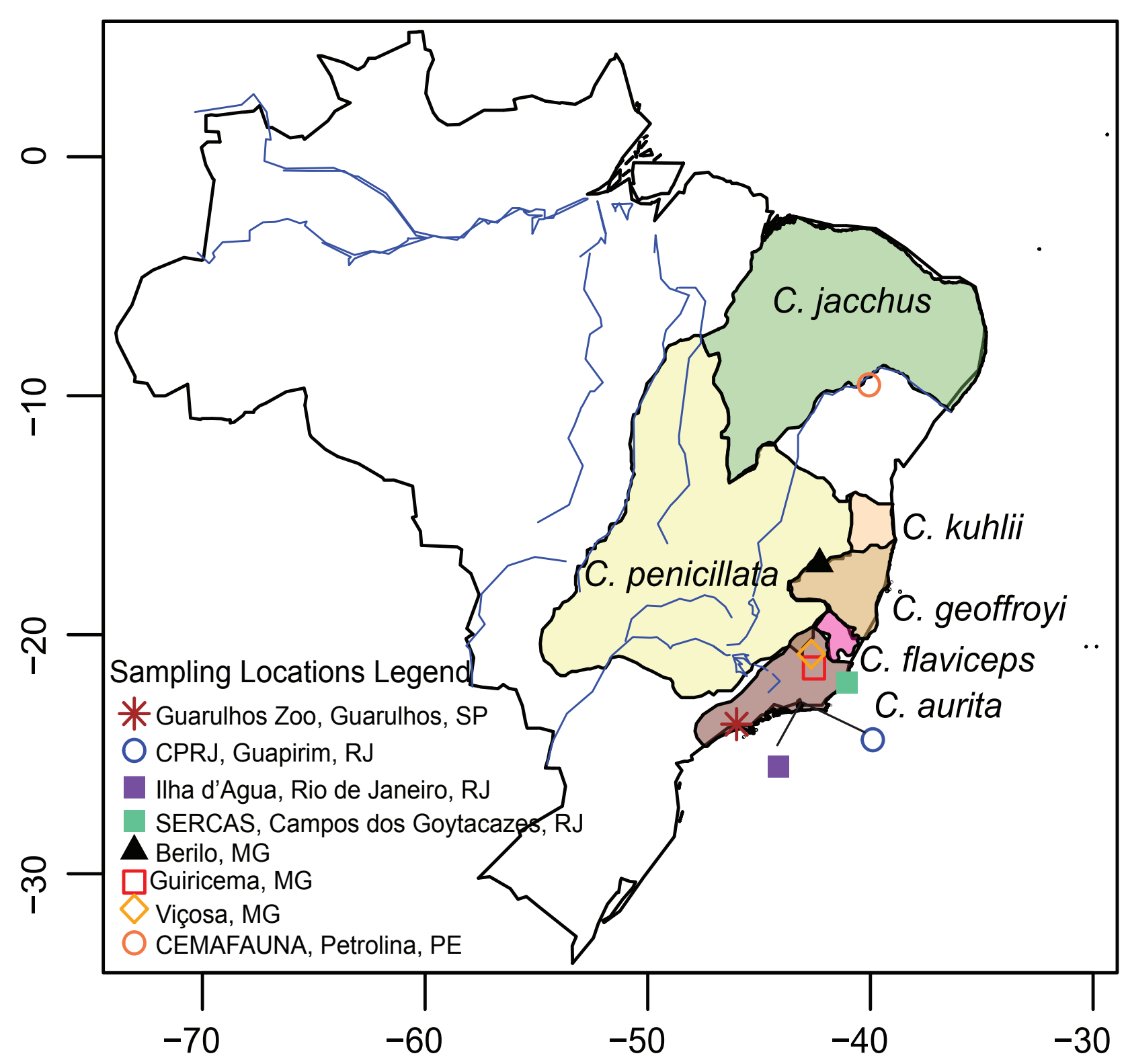

Figure 1. Natural Callithrix ranges and sampling locations in Brazil. Sampling locations are represented by different colored shapes and species names are written next to their respective ranges. Ranges are based on 2012 IUCN Red List Spatial Data from http://www.iucnredlist.org/technical-documents/spatial-data. Legend abbreviations are as follows- MG: Minas Gerais, Rio de Janeiro: RJ, Pernambuco: PE, São Paulo: SP; CPRJ: Centro de Primatologia do Rio de Janeiro; CEMAFAUNA: Centro de Conservação e Manejo da Fauna da Caatinga. 
bioRxiv preprint doi: https://doi.org/10.1101/708255; this version posted July 28,2021 . The copyright holder for this preprint (which was not certified by peer review) is the author/funder. All rights reserved. No reuse allowed without permission.

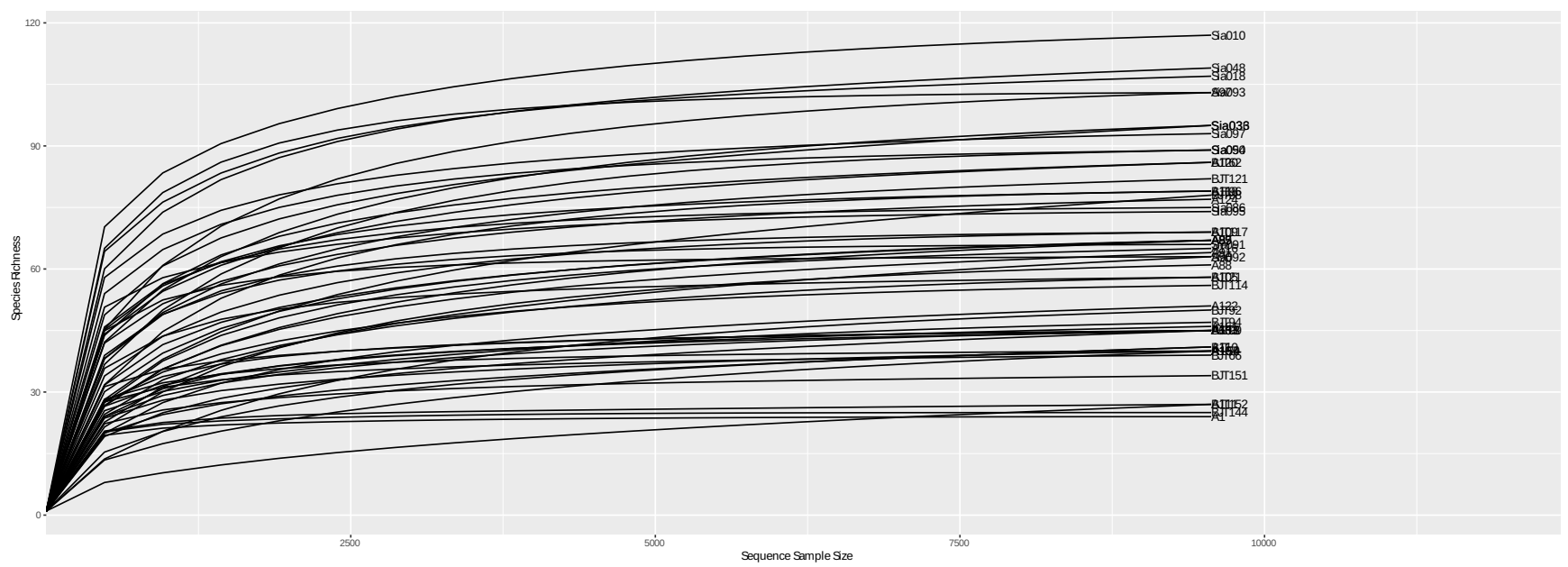

Figure 2. Sample rarefaction curve after rarefying for the sample with the lowest sequencing depth. 

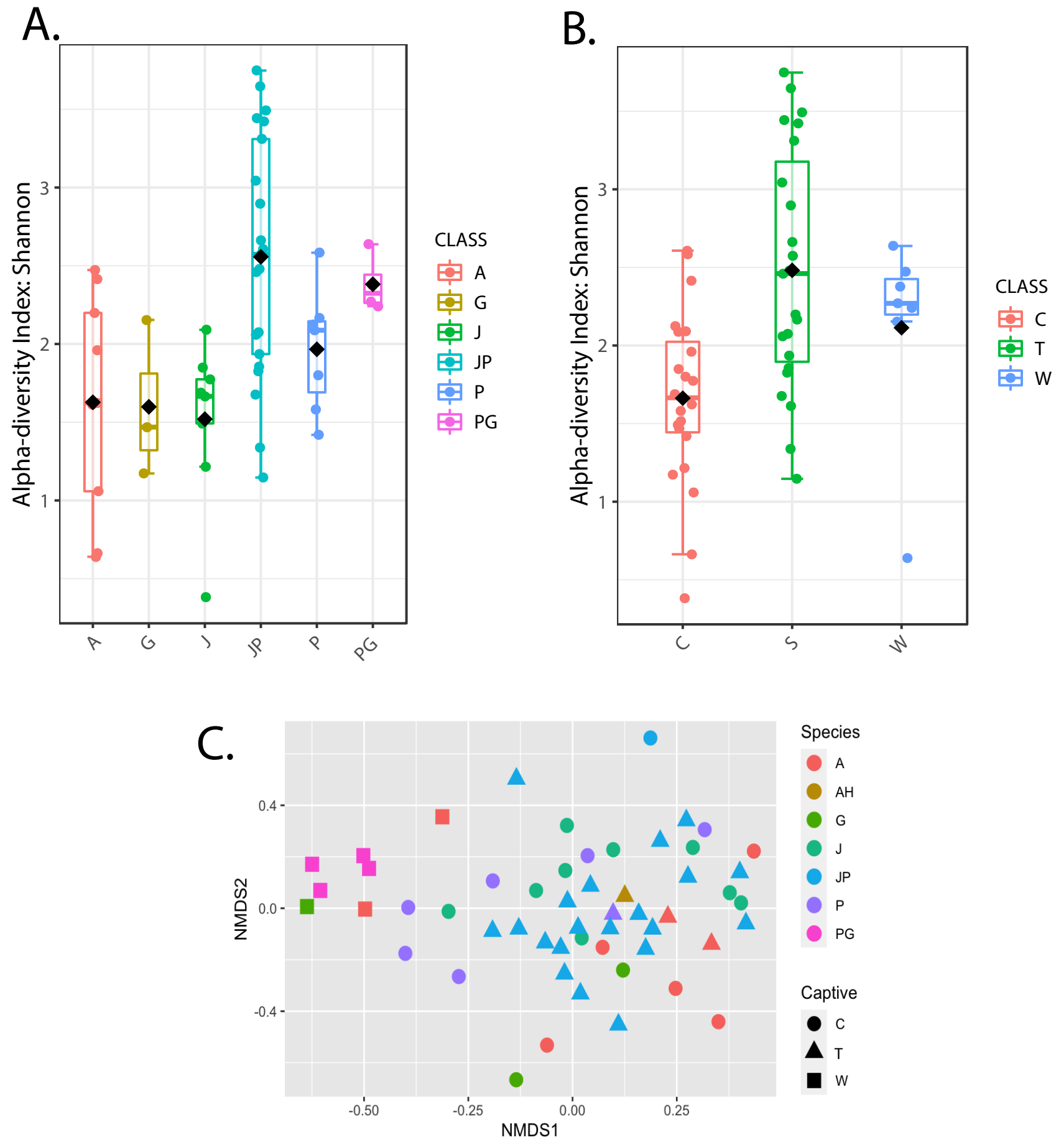

Figure 3. Boxplots of gut microbiome species alpha diversity Shannon diversity index by host taxon (A) and Shannon diversity index by host environment (B). Classifications along the $\mathrm{x}$-axis in panels $\mathrm{A}$ are $\mathrm{A} / \mathrm{Red}=C$. aurita, $\mathrm{G} / \mathrm{Yellow}-\mathrm{Green}=C$. geoffroyi, J/Green= C. jacchus, P/Blue C. penicillata, JP/Blue-Green=C. jacchus $\times$ C. penicillata hybrids, and PG/Pink=C. penicillata $\times$ C. geoffroyi hybrids. Classifications along the $\mathrm{x}$-axis in panels B are C/Red=Captive, T/Green=Translocated, and W/Blue=Wild. Panel (C) shows an NMDS ordination plot for gut microbiome beta-diversity measured by the Bray-Curtis dissimilarity index. Host species classifications in panel $\mathrm{C}$ are $\mathrm{A} / \mathrm{Red}=$ C. aurita, $\mathrm{G} /$ Yellow-Green=C. aurita $\mathrm{x}$ Callithrix sp. hybrid, G/Hunter Green= C. geoffroyi J/Light Green=C. jacchus, JP/Blue-Breen=C. jacchus $\times$ C. penicillata hybrids, P/Purple C. penicillata, and PG/Pink=C. penicillata $\mathrm{x}$. geoffroyi hybrids. Host environment classifications in panel $\mathrm{C}$ are classified by Circle/C=Captive, Triangle/T=Translocated, and Square/W=Wild. 
bioRxiv preprint doi: https://doi.org/10.1101/708255; this version posted July 28,2021 . The copyright holder for this preprint (which was not certified by peer review) is the author/funder. All rights reserved. No reuse allowed without permission.

A.

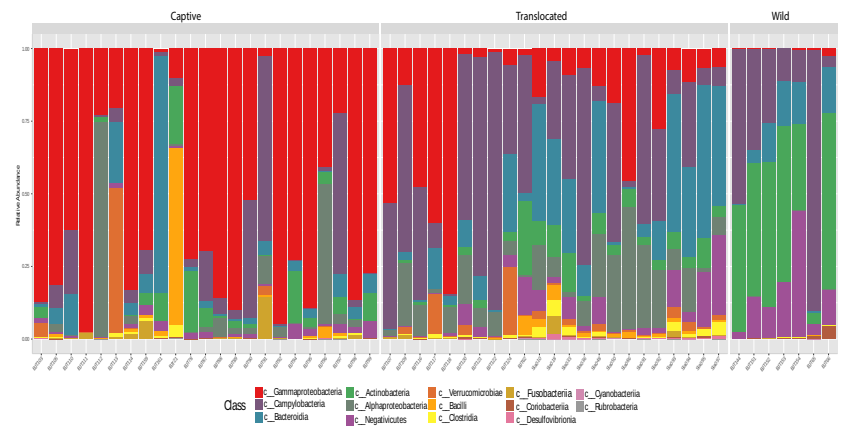

B.

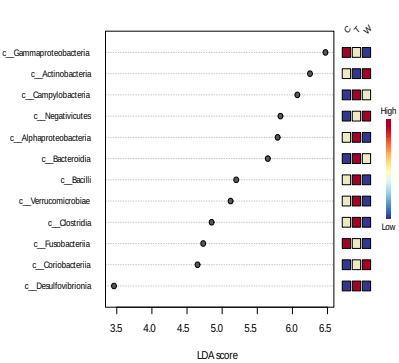

C.

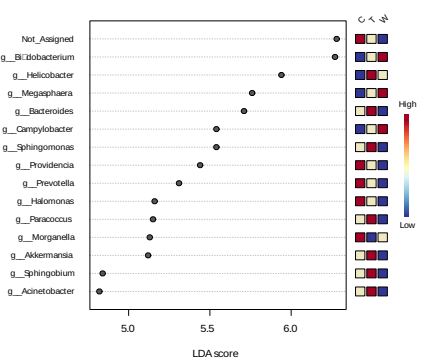

Figure 4. (A). Relative levels of class level bacterial abundance by host environment classification (B). LefSe analysis of bacterial class abundance categorized by host environment. (C). LefSe analysis of bacterial genus abundance categorized by host environment. Host environment classifications in plots are $\mathrm{C}=$ Captive, $\mathrm{T}=$ Translocated, and $\mathrm{W}=$ Wild. 
A.

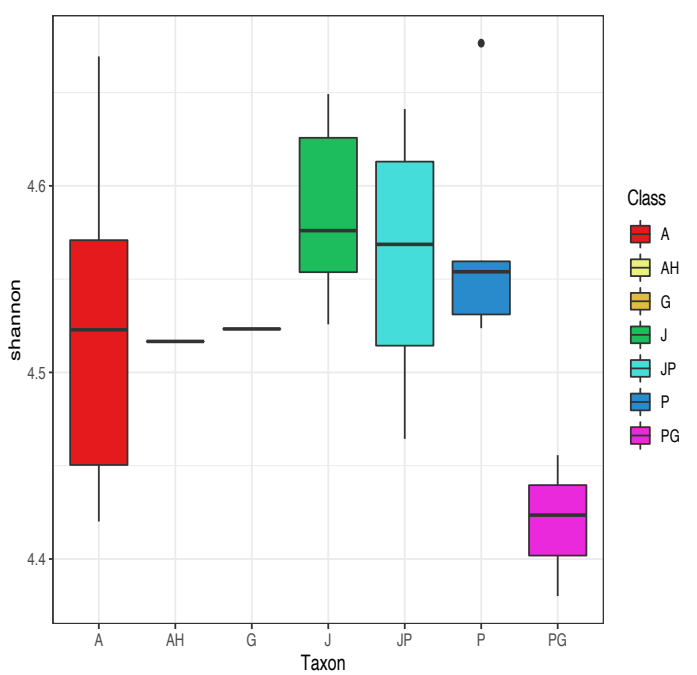

B.

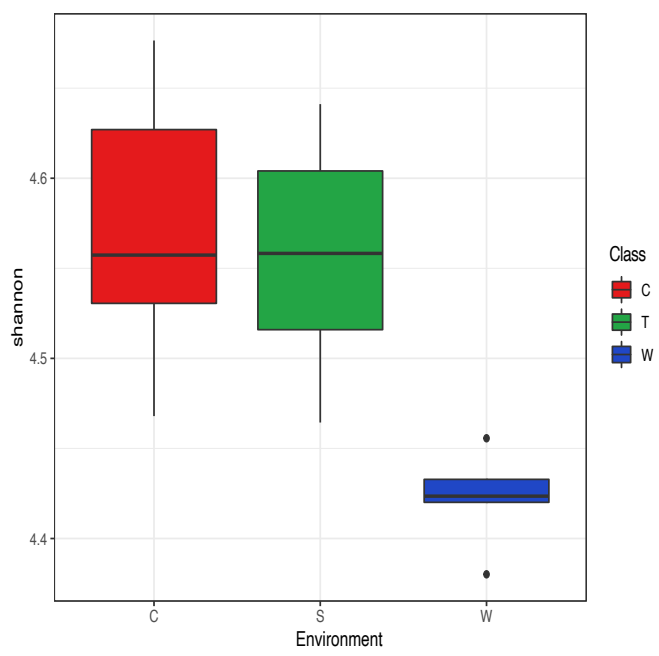

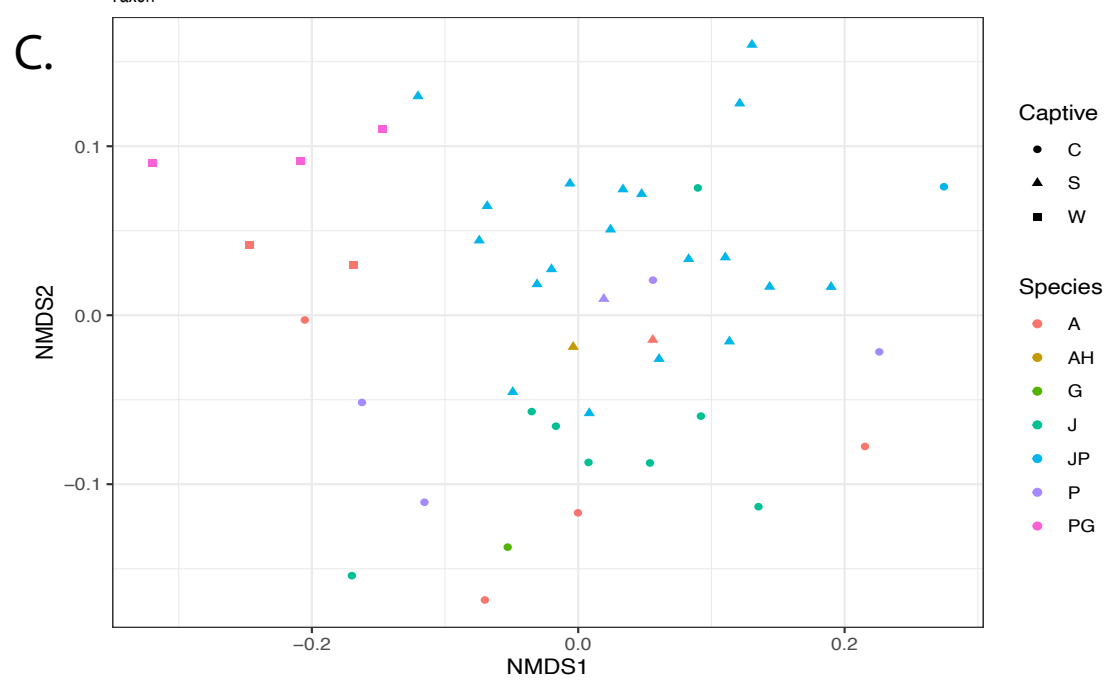

Figure 5. Boxplots of gut microbiome predicted gut KEGG pathways alpha diversity Shannon diversity index by host taxon (A) and Shannon diversity index by host environment (B). Classifications along the $\mathrm{x}$-axis in panels $\mathrm{A}$ is $\mathrm{A} / \mathrm{Red}=C$. aurita, $\mathrm{G} /$ Yellow-Green $=C$. geoffroyi, $\mathrm{J} / \mathrm{Green}=C$. jacchus, $\mathrm{P} / \mathrm{Blue} C$. penicillata, $\mathrm{JP} / \mathrm{Blue}-\mathrm{Green}=C$. jacchus $\mathrm{x} C$. penicillata hybrids, and $\mathrm{PG} / \mathrm{Pink}=C$. penicillata $\mathrm{x}$ C. geoffroyi hybrids. Classifications along the $\mathrm{x}$-axis in panels B are C/Red=Captive, T/Green=Translocated, and W/Blue=Wild. Panel (C) shows an NMDS ordination plot for gut microbiome predicted gut KEGG pathways beta-diversity measured by the Bray-Curtis dissimilarity index. Host species classifications in panel $\mathrm{C}$ are $\mathrm{A} / \mathrm{Red}=C$. aurita, G/Yellow-Green=C. aurita $\mathrm{x}$ Callithrix sp. hybrid, G/Hunter Green=C. geoffroyi $\mathrm{J} /$ Light Green= . jacchus, JP/Blue-Breen $=C$. jacchus $\times$ C. penicillata hybrids, $\mathrm{P} /$ Purple $C$. penicillata, and PG/Pink=C. penicillata $\times$. geoffroyi hybrids. Host environment classifications in panel $\mathrm{C}$ are classified by Circle/C=Captive, Triangle/T=Translocated, and Square/W=Wild. 
A.

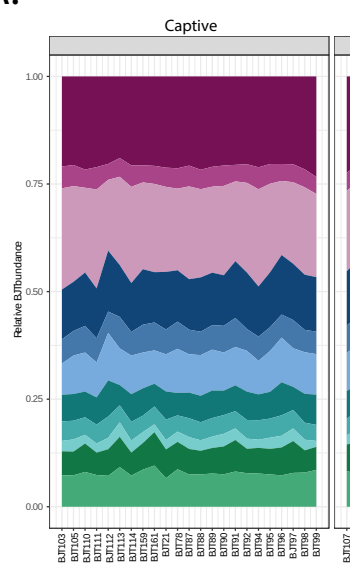

Translocated

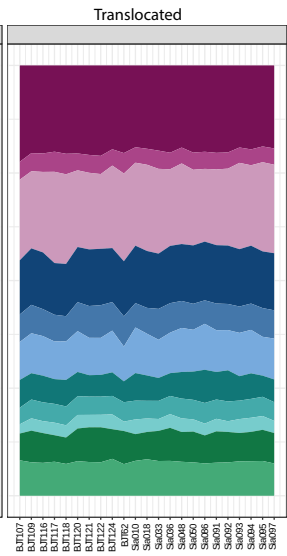

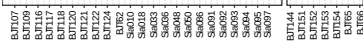

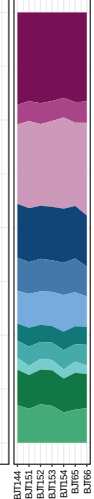

Wild

C.

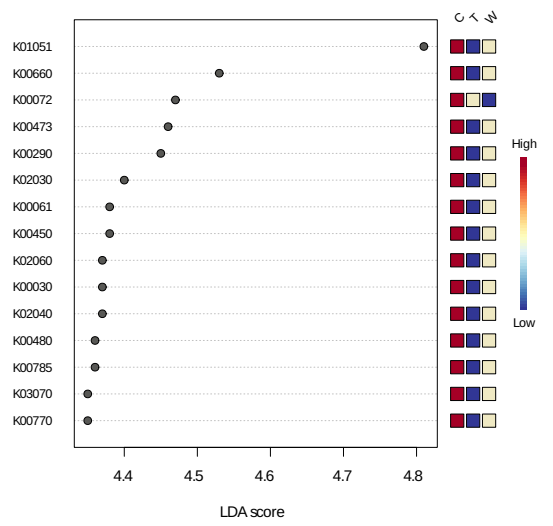

B.
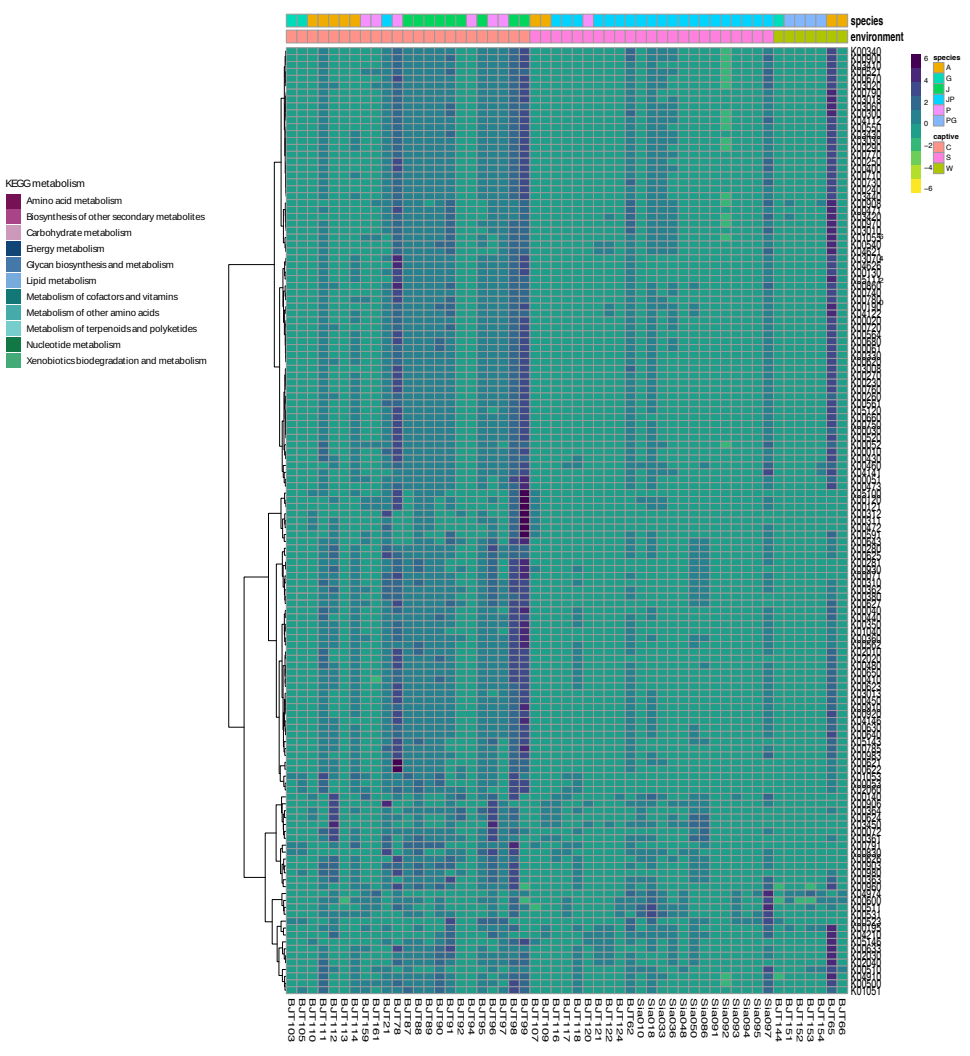

Figure 6. (A). Relative abundance of predicted KEGG pathways by host environment classification (C). LefSe analysis of predicted KEGG pathway abundance by host environment. LefSe analysis of bacterial taxa abundance by host environment at genus level. Host environmental classifications in plots are $\mathrm{C}=$ Captive, $\mathrm{T}=$ Translocated, and $\mathrm{W}=\mathrm{Wild}$. 
A.

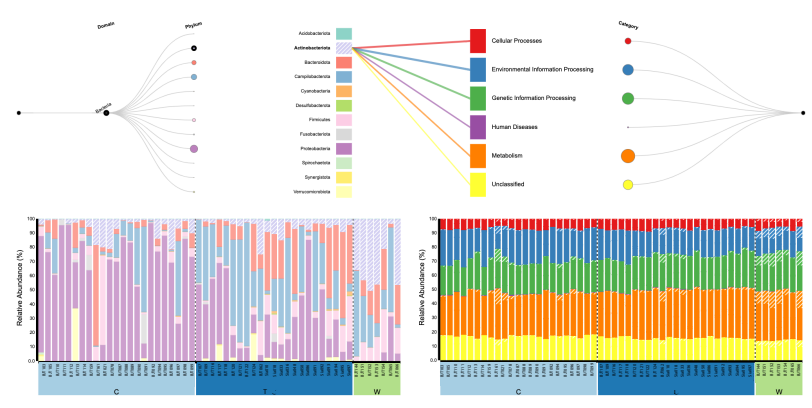

c.
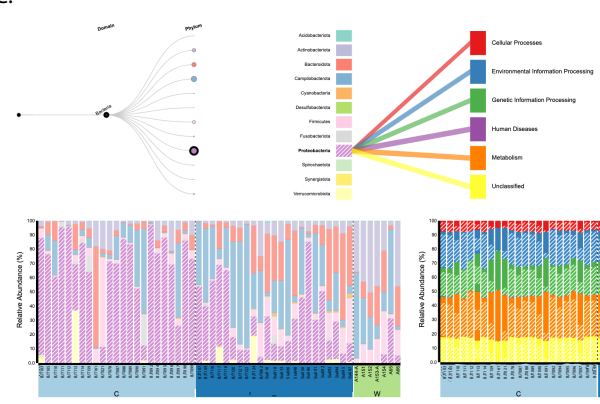

B.

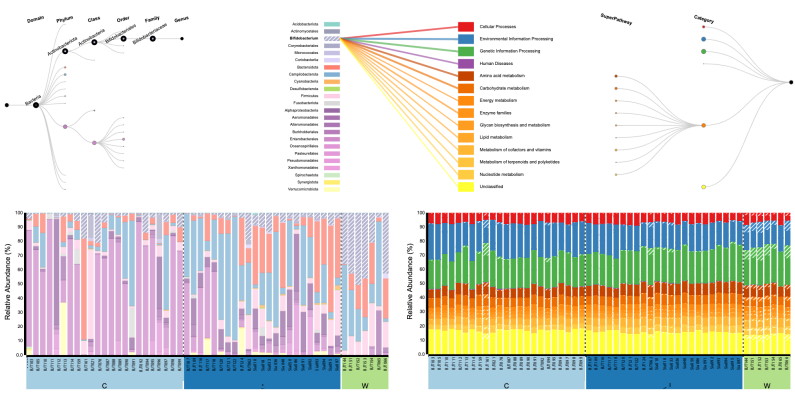

D.
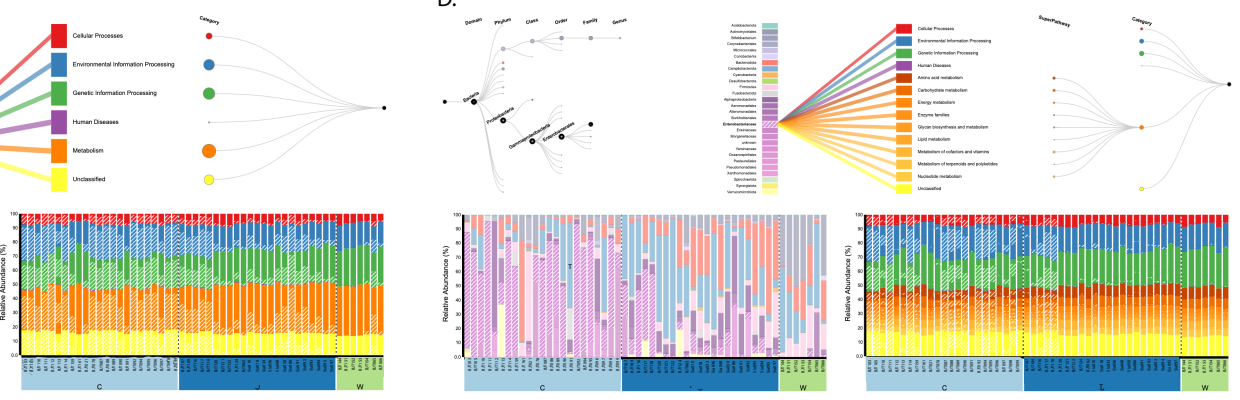

Figure 7. Visualization of BURRITO results showing linkage between Callithrix gut bacterial taxa composition and predicted functional profiles In each plot, the upper left corner shows bacterial phylogeny, the lower left corner shows bacterial taxa relative abundance, the upper right shows a network of functional processes, and the lower right shows predicted relative abundances of major functional categories of the Callithrix gut. The middle upper portion of each plot shows distribution of involvement of specific bacterial taxa in functional processes. Thickness of connecting lines between bacterial classes and functional classes indicates stronger involvement of a given bacterial taxon in a given functional process. The position of bacterial taxa and functional processes among respective relative abundance plots is represented by diagonal stripes. Host environment classifications in all plots are classified by $\mathrm{C}=$ Captive, $\mathrm{T}=$ Translocated, and $\mathrm{W}=\mathrm{Wild}$. (A). Distribution of Actinobacteria role in predicted functional processes. (B) Distribution of Bifibacterium role in predicted functional processes, with expansion of metabolic processes. (C) Distribution of Proteobacteria role in predicted functional processes. (D) Distribution of Enterobacteriaceae role in predicted functional processes, with expansion of metabolic processes. 